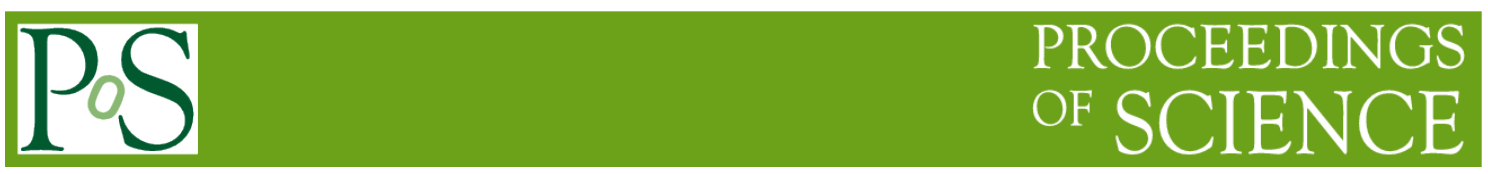

\title{
Realistic Texture Reconstruction Based on Associated Mapping
}

\section{Zicai Liu'}

PLA University of Science and Technology, Nanjing, 210000, China

E-mail: $463711687 @$ qq. com

\section{Guyu $\mathrm{Hu}^{2}$}

PLA University of Science and Technology, Nanjing, 210000, China

E-mail: 513899463 @qq. com

\section{Jianxin Luo ${ }^{3}$}

PLA University of Science and Technology, Nanjing, 210000, China

E-mail: lacp7723412@163.com

In the virtual reality scene, the texture reconstructionprocess of real model is extremely complex. Generally, it requires integrating a large number of images with Most of these images associated with one or more of them. Hereby, an algorithm to extract the perspective projection matrix based on calibration method and an algorithm to construct associated view matrix based on feature matching are presented in this paper. In combination with these two algorithm, the realistic texture can be reconstructed by the Projection Texture Mapping. By this method, the complicated reconstruction process can be simplified. Experimental results show that this method is stable, practical and effective in the reconstruction of realistic texture.

ISCC 2015

18-19, December 2015

Guangzhou, China

\section{${ }^{1} \mathrm{ZiCai}$ Liu}

${ }^{2} \mathrm{GuYu} \mathrm{Hu}$

${ }^{3}$ Corresponding Authors: Jianxin Luo

This study is supported by National 863 Plan Project (2012AA01A509) and Youth Science Fund Project of Jiangsu Province (BK20150722) 


\section{Introduction}

In 3D modeling, the texture mapping[1-2] is an important part of realistic graphics. While using texture mapping, we can produce vivid realistic graphics expediently. At present, the primary texture manufacture is:

(1) Photoshop manufacture; but the fusion of multiple knowledge and the requirements of advanced technology increase the difficulty of manufacturing.

(2) Splicing manufacture; but the corner of the splicing images may have deformation that will affect the texture coordinate orientation.

The difficulty of the texture manufacture often stuck people into trouble; however, the texture mapping is an indispensable existence to the model reconstruction, especially the realistic texture integrated by the realistic images. Nevertheless, if we can find out the association between these images, the efficiency of reconstruction will be greatly improved.

Based on the said requirements, this paper presents a realistic texture reconstruction algorithm on the basis of association mapping. In this method, the realistic texture is reconstructed by Projection Texture Mapping combined with Calibration and feature Matching[3-4]. Experiments show that the realistic texture reconstruction algorithm based on associated mapping highlights sound effect in the application while it can effectively improve the efficiency of rendering; furthermore it has the great fidelity and adaptability.

\section{Related Works}

The texture location and deformation have always been important problems for a long time. All the scholars are trying to find out a cost-effective way to solve the location and deformation problems.

In recent researches, Model Reconstruction of 3D Object Surface Texture used the turning device to locate the texture and used the several-angle texture mapping to avoid the deformation[5]. Obviously, the turning device is not suitable for practical application. Though the several-angle texture mapping is effective, the acquisition and selection of texture is too complex for rendering.

In Rapidly 3D Texture Reconstruction Based on Oblique Photography[6], they locate the texture by collinear equation calculation and texture association based on aerial trajectory. This location method is convenient and accurate; but its high cost is not suitable for all application scenarios, moreover, they avoid the deformation by texture rectification and vectors of model grid;however, the large number of vectors will cost too much time in computing.

\section{Projective Texture Mapping}

The Projective Texture Mapping is frequently used in the volume rendering algorithm and the shadow algorithm[7]. In this paper, using Projective Texture Mapping is combined with calibration and feature matching. This combination makes the Projective Texture Mapping more practical; furthermore, the adaptability of projective texture mapping can decrease the distortion of realistic texture reconstruction. The mapping process is shown as follows:

(1) transform the vertex's world coordinate to the eye coordinate by view matrix.

(2) transform the vertex's eye coordinate to the projection space by projection matrix.

(3) as the normalized projection space is $[-1,1]$ and the coordinates of the texture space is

$[0,1]$. Thus we need to convert the projection space to the texture space by translation matrix.

In this sense, the transformation matrix of Projective Texture Mapping is shown as follows: 


$$
M=\left[\begin{array}{cccc}
0.5 & 0 & 0 & 0.5 \\
0 & 0.5 & 0 & 0.5 \\
0 & 0 & 0.5 & 0.5 \\
0 & 0 & 0 & 1
\end{array}\right] P V
$$

\section{Extraction Algorithm of Projection Matrix Based on Calibration}

The Projective Texture Mapping has the advantages of simple process, fast rendering and significant effect; however, the extraction of projection matrix is always a problem. The traditional extraction is manual measurement and self-calibration of advanced camera. This is one of the important reasons for the limitation of projection texture mapping. Hence, the extraction algorithm of projection matrix based on calibration is presented to make it possible to extract the projection matrix from the image taken by the general camera.

\subsection{Extract Parameter Matrix by Calibration of Zhang}

Calibration of Zhang is a new method of extracting the camera internal and external parameters based on the radial distortion problem[8]. Because of its easy usage, low cost, good robustness and high accuracy, Calibration of Zhang has been widely used.

Theoretical derivation and practical work have proved the following relationship between the image plane and the three-dimensional space:

$$
s\left[\begin{array}{l}
u \\
v \\
1
\end{array}\right]=A\left[\begin{array}{ll}
R & T
\end{array}\right]=A\left[\begin{array}{llll}
r_{1} & r_{2} & r_{3} & t
\end{array}\right]\left[\begin{array}{c}
X_{w} \\
Y_{w} \\
Z_{w} \\
1
\end{array}\right]
$$

Where $\mathrm{A}$ is the parameter matrix, $\mathrm{R}$ is the rotation matrix, $\mathrm{t}$ is the translation vector and $\mathrm{s}$ is a scalar zoom factor. The goal of the calibration is to extract the matrix A.

Let the Z-plane of world Coordinate system be located on the plane of calibration target plane. Then we will have $Z_{w}=0$. Then, Equation (4.1) can be transformed into:

$$
s m=H M
$$

Where $H=A\left[\begin{array}{lll}r_{1} & r_{2} & t\end{array}\right]=\left[\begin{array}{lll}h_{1} & h_{2} & h_{3}\end{array}\right]=\left[\begin{array}{lll}h_{11} & h_{12} & h_{13} \\ h_{21} & h_{22} & h_{23} \\ h_{31} & h_{32} & h_{33}\end{array}\right], \quad M=\left[\begin{array}{c}X_{w} \\ Y_{w} \\ Z_{w} \\ 1\end{array}\right], \quad m=\left[\begin{array}{c}u \\ v \\ 1\end{array}\right]$

The corner point $\mathrm{m}$ of chess board in the image plane can be obtained by Harris Corner Detector[9]. And the corresponding point $\mathrm{M}$ in world Coordinate system can be obtained by the standard chess board. Thus we can figure out a matrix $\mathrm{H}$ for each image.

Because $\mathrm{r}_{1}$ and $\mathrm{r}_{2}$ are orthogonal, we can get $\mathrm{r}_{1}^{\mathrm{T}} \mathrm{r}_{1}=\mathrm{r}_{2}^{\mathrm{T}} \mathrm{r}_{2}=1$ and $\mathrm{r}_{1}^{\mathrm{T}} \mathrm{r}_{2}=0$. So:

$$
\begin{gathered}
h_{1}^{T} A^{-T} A^{-1} h_{2}=0 \\
h_{1}^{T} A^{-T} A^{-1} h_{1}=h_{2}^{T} A^{-T} A^{-1} h_{2} \\
B=A^{-T} A^{-1}=\left[\begin{array}{lll}
B_{11} & B_{12} & B_{13} \\
B_{21} & B_{22} & B_{23} \\
B_{31} & B_{31} & B_{33}
\end{array}\right]
\end{gathered}
$$

Set

Matrix B is a symmetric matrix, so it can be written in the following form : 


$$
b=\left[\begin{array}{llllll}
B_{11} & B_{12} & B_{22} & B_{13} & B_{13} & B_{33}
\end{array}\right]
$$

Set the column vector of matrix $\mathrm{H}$ be represented as:

$$
h_{i}=\left[\begin{array}{lll}
h_{i 1} & h_{i 2} & h_{i 3}
\end{array}\right]
$$

According to Equation (4.6), Equation (4.4) can be rewritten as follows:

$$
h_{i}^{T} B h_{i}=V_{i j}^{T} b
$$

Where

$$
V_{i j}=\left[\begin{array}{lllll}
h_{i 1} h_{j 1} & h_{i 1} h_{j 2}+h_{i 2} h_{j 1} h_{i 2} h_{j 2} \quad h_{31} h_{j 1}+h_{i 1} h_{j 3} h_{31} h_{j 1}+h_{i 3} h_{j 3} h_{i 3} h_{i 3}
\end{array}\right]
$$

With Equation (4.8), Equations (4.4) can be rewritten as:

$$
\left[\begin{array}{c}
V_{12}^{T} \\
V_{12}^{T}-V_{22}^{T}
\end{array}\right] b=0
$$

According to Equation (4.9), it can set up two equations with six unknown to each image. With the knowledge of algebra, it will need at least six equations to work out six unknowns, which means it will need at least three images to figure out Matrix B. With Matrix B, we can get Matrix A by Equation (4.4).

The solving process above is nonlinear optimized; therefore, it will be essential of optimization by Levenberg-Marquarat [10]. After that, we can work out the wanted Matrix A:

$$
A=\left[\begin{array}{ccc}
f / d_{x} & 0 & u_{0} \\
0 & f / d_{y} & v_{0} \\
0 & 0 & 1
\end{array}\right]
$$

Where $\mathrm{f}$ is the focal length. $\mathrm{d}_{\mathrm{x}}$ and $\mathrm{d}_{\mathrm{y}}$ is the pixel Size. $\mathrm{u}_{0}$ and $\mathrm{v}_{0}$ is the center of image.

\subsection{Extraction Algorithm of Projection Matrix}

In principle, the parameter matrix and the projection matrix are the same transition matrix which can convert the three-dimensional space into the plane space. The extraction algorithm of projection matrix is used to extract the projection matrix from parameter matrix.

The projection matrix is as follows:

$$
P=\left[\begin{array}{cccc}
\frac{1}{r} \cot \left(\frac{a}{2}\right) & 0 & 0 & 0 \\
0 & \cot \left(\frac{a}{2}\right) & 0 & 0 \\
0 & 0 & \frac{-(F+N)}{F-N} & \frac{-2 \mathrm{FN}}{F-N} \\
0 & 0 & -1 & 0
\end{array}\right]
$$

Where $\mathrm{r}$ is the aspect ratio, $\mathrm{a}$ is the field angle, $\mathrm{F}$ is the far clipping plane and $\mathrm{N}$ is the near clipping plane.

From Equation (4.10), the horizontal and longitudinal viewing angle can be obtained:

$$
\partial=\frac{f / d_{x}}{m / 2} \quad \beta=\frac{f / d_{y}}{n / 2}
$$

Where $\mathrm{m}$ and $\mathrm{n}$ is the height and width of image. Then we can get the aspect ratio:

$$
r=\frac{\beta}{\partial}=\frac{m d_{x}}{n d_{y}}
$$


Because of the projection is independent of $\mathrm{F}$ and $\mathrm{N}, \frac{-(F+N)}{F-N}$ and $\frac{-(F+N)}{F-N}$ can be set as 1 . The wanted projection matrix is:

$$
P=\left[\begin{array}{cccc}
\frac{1}{r} \cot \left(\frac{a}{2}\right) & 0 & 0 & 0 \\
0 & \cot \left(\frac{a}{2}\right) & 0 & 0 \\
0 & 0 & 1 & 1 \\
0 & 0 & -1 & 0
\end{array}\right]
$$

\section{Construction Algorithm of Associated View Matrix based on Feature Matching}

Most images of the reconstruction are associated with one or more of them; therefore, the aim of the construction algorithm is to get the associated view matrix through the association obtained by SIFT feature extraction and matching.

\subsection{SIFT Feature Selection base on RANSAC Algorithm}

SIFT feature is one of the most representative algorithm in respect of the feature detection[11]. It has three advantages: first, stable detection of the feature location; secondly, scale invariance and rotation invariance; third, tolerance of light, noise and change of micro perspective. SIFT feature selection base on RANSAC algorithm consists of three parts:

First, SIFT feature detection. It mainly includes the following four steps:

(1) Detection of the extreme point in scale space

(2) Precise position of the extreme point

(3) Specification of the feature point's direction

(4) Generation of the feature vectors

Second, SIFT features matching. It uses the Euclidean distance as the measurement of the matching. The matching process is as follows: choose a non-matching feature point in reference image; then find out two feature points which have the shortest Euclidean distance in associated image. If the specific value of these two feature points is less than the threshold value, the matching should be retained. We can get a set of matching by operating to all feature points.

Third, wipe off the wrong match by RANSAC algorithm:

(1) Get a sample with four group matching randomly for RANSAC algorithm[12]

(2) Figure out the transformation matrix $\mathrm{H}$ of the samples above

(3) According to the sample set, transformation matrix and error indicator, identify the appropriate matching points and add it to the consistent set $\mathrm{s}$.

(4) According to the element's quantity of s, estimate whether it is the optimal consistent set S. Update the set S.

Update the error probability $p$,accept the optimal consistent set $S$ if $p$ is less than threshold value. Otherwise, repeat Step (1) to (4) until p meets the demands.

\subsection{Construction Algorithm of Associated View Matrix}

The associated view matrix's construction is the key technique of the realistic texture reconstruction based on associated mapping. Since the effect of view matrix is same as [R T], we can change Equation (4.1) into: 


$$
s v_{s}=S\left[\begin{array}{c}
u \\
v \\
1
\end{array}\right]=A\left[\begin{array}{ll}
R & T
\end{array}\right]\left[\begin{array}{c}
X_{w} \\
Y_{w} \\
Z_{w} \\
1
\end{array}\right]=A V\left[\begin{array}{c}
X_{w} \\
Y_{w} \\
Z_{w} \\
1
\end{array}\right]
$$

According to Equation (5.2), we have:

$$
s v_{s 1}=A V_{1} v_{w} \quad s v_{s 2}=A V_{2} v_{w}
$$

Where $\mathrm{v}_{\mathrm{s} 1}$ and $\mathrm{v}_{\mathrm{s} 2}$ are the matching group of $\mathrm{S}$. They have the relationship as follows:

$$
v_{s 2} H=v_{s 1}
$$

Next, we need to construct the associated view matrix with $A, V_{1}, v_{s 1}$ and $v_{s 2}$.

According to Equation (5.3) and (5.4), we have:

$$
v_{w}=V_{1}^{-1} A^{-1} v_{s 1} \quad A V_{2} v_{w} H=A V_{1} v_{w}
$$

Where $\mathrm{s}$ is a constant which can be omitted. Then the associated view matrix $\mathrm{V}_{2}$ can be deduced from Equation (5.5):

$$
V_{2}=A^{-1} v_{s 2} v_{s 1}^{-1} A V_{1}
$$

With the associated view matrix $\mathrm{V}_{2}$, we can get the transformation matrix of associated projection texture mapping:

$$
M^{\prime}=\left[\begin{array}{cccc}
0.5 & 0 & 0 & 0.5 \\
0 & 0.5 & 0 & 0.5 \\
0 & 0 & 0.5 & 0.5 \\
0 & 0 & 0 & 1
\end{array}\right] P A^{-1} v_{s 2} v_{s 1}^{-1} A V_{1}
$$

\subsection{Realistic Texture Reconstruction Based on Associated Mapping}

The associated object of the texture images is uncertain; Hence, we should get the associated texture first:

(1) Based on reference texture, get the association set 1 and the non-associate set; at meanwhile, record the associated texture

(2) Based on the associated packs 1, get the association set 2 and the non-associate set; at meanwhile, record the associated texture

(3) Repeat step (1) to (2) until the non-associate set has no association with the current association set. Then, we can get all the associated texture

(4) Delete the rest of non-affiliated texture

After all the collection of associated texture, we can reconstruct the realistic texture from the reference texture. The main process is shown as follows:

(1) Map the reference texture

(2) Based on the reference texture, map the texture in Set 1 by the associated view matrix between reference texture and texture in Pack 1

(3) Construct the associated view matrix between texture in Set 1and Set 2,and then map the texture in Set 2

(4) Do the same operation until all the textures have been mapped

After the pretreatment of feature detection and matching, we can realize the accurate location and fast associated texture mapping. 


\section{Results and Analysis}

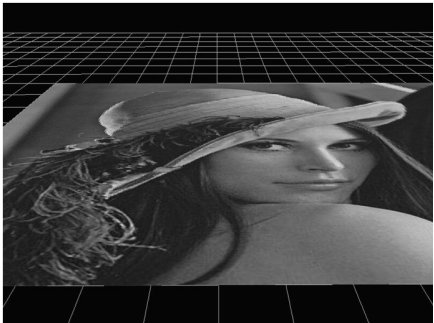

(a) Reference Texture

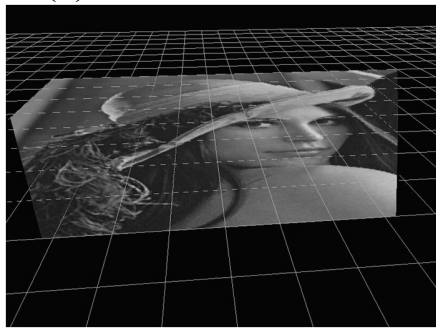

(d) Reference mapping results

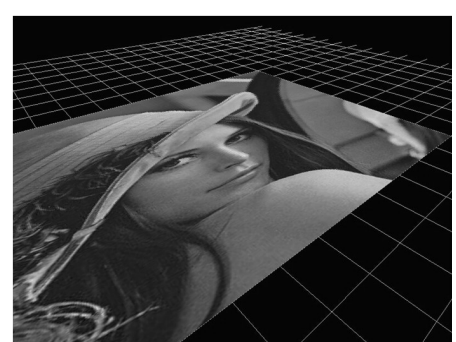

(b) Associated Texture

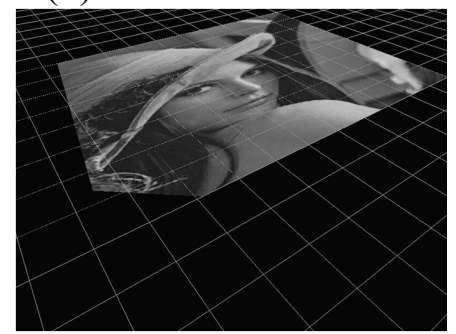

(e) Association mapping results

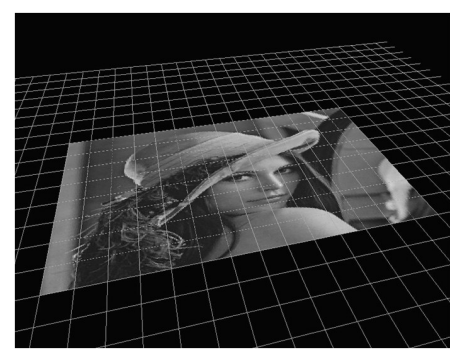

(c)Scene Graph

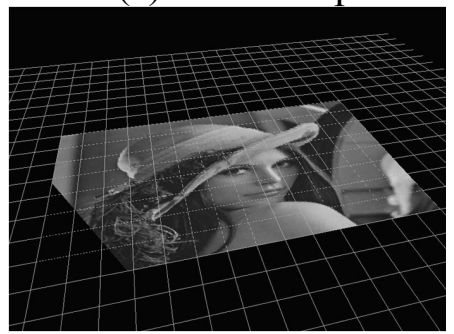

(f)Reconstructed Results

Figure 1: Experimental Result

The reconstruction results of Lena in virtual scene is shown in Fig. 1. Texture (a) and (b) each contains part of Lena. Mapping (b) based on (a), we can get Fig. (e). Displaying (d) and (e) at the same time in the virtual scene, we can get the reconstruction (f).

As we can see, the reference texture (a) lacks the information of left bottom. Also the reconstruction (f) lacks the left bottom. Compared with the scene graph (c), the position of Lena in Fig. (f) is very accurate.

\begin{tabular}{|c|c|c|c|}
\hline Field angle & $60^{\circ}$ & $90^{\circ}$ & $120^{\circ}$ \\
\hline Average error & $0.5562^{\circ}$ & $0.7896^{\circ}$ & $1.3153^{\circ}$ \\
\hline
\end{tabular}

Figure 2: Error between extracted field angle and accurate field angle

Tested by experiments. The error in Fig. 2 is acceptable for the experiment.

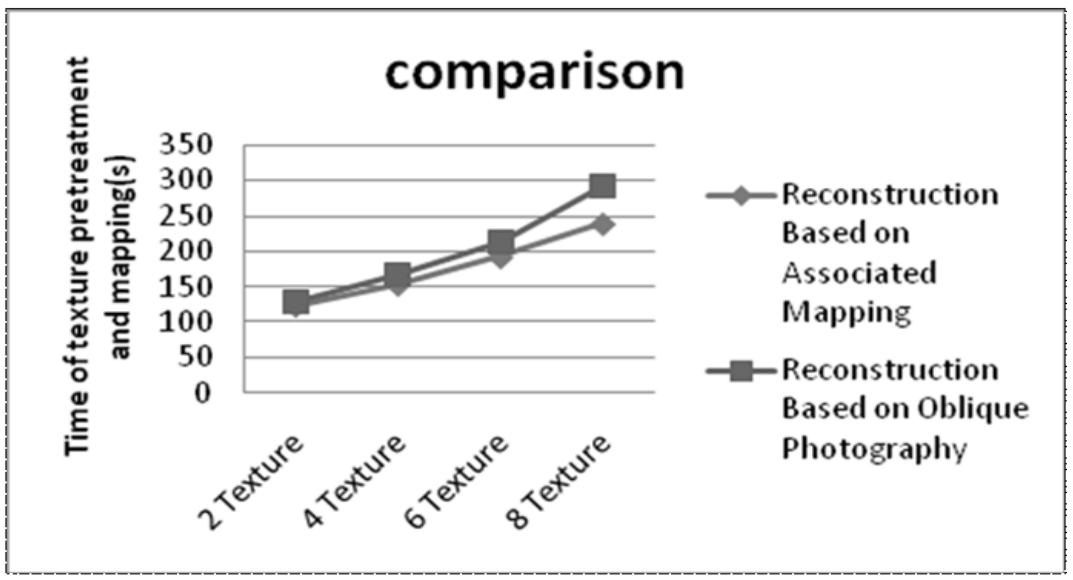

Figure3: Compared with the splicing reconstruction

Fig. 3 shows that realistic texture reconstruction based on associated mapping is more efficient than the reconstruction based on the Oblique photography.

In order to make the location method more suitable, this paper figures out the transformation relationship between the associated texture and reference texture, which means it 
can locate the texture based on the reference texture without any assistance of external device; at meanwhile, the Projection Texture Mapping renders the texture after the model converts to view-frustum so that it can avoid the deformation caused by model grid. Furthermore, without the calculation of model grid, the rendering efficiency will be greatly improved.

\section{Summary}

In this paper, in order to simplify the process, we use Projective Texture Mapping in combination with Calibration and SIFT feature to reconstruct the realistic texture. The advantage of this method is that we can locate the texture accurately by means of the association analysis; moreover, we can improve the efficiency by Projective Texture Mapping, however, the multiple rendering of public area will affect the efficiency. Besides, the texture junction of reconstruction model is defective. Thus we will try to reduce duplication of rendering and perfect the reconstruction in the future.

\section{References}

[1] M. Favorskaya, M. Damov, A . Zotin. Intelligent Texture Reconstruction of Missing Data in Video Sequences Using Neural Networks[M]. Advanced Techniques for Knowledge Engineering and Innovative Applications, Springer Berlin Heidelberg.pp, 163-176 (2013).

[2] P. V. Sander, J. Snyder, S. J. Gortler. Texture mapping progressive meshes [J]. Siggraph, 35(7):409416(2001).

[3] D. Jia. Algorithm Research of Camera Calibration [D].Harbin: Harbin Engineering University (2007).(In Chinese)

[4] I. B. Fidaner, A.T. Cemgil. Summary Statistics for Partitionings and Feature Allocations[J]. Eprint Arxiv. 23(2) 261-269 (2013).

[5] L. L. Dai, L. J. Ding. Model reconstruction of 3D object surface texture [J]. Journal of Jiang su University (Natural Science Edition ). 27(5): 21-23(2006). (In Chinese)

[6] C. S. Zhang, W. L. Zhang. Rapidly 3D Texture Reconstruction Based on Oblique Photography [J]. Acta Geodaetica et Cartographica Sinica. 44(7): 782-780.(2015)(In Chinese)

[7] C. Everitt. Projective texture mapping [M]. Nvidia Technical Report, California. pp, 1-7(2001).

[8] Z. Zhang. A Flexible New Technique for Camera Calibration [J]. Pattern Analysis \& Machine Intelligence IEEE Transactions on. 22(11):1330-1334(2000).

[9] J. B. Ryu, C. G. Lee, H. H. Park. Formula for Harris corner detector [J]. Electronics Letters. 47(3):180-181(2011).

[10] A. Ranganathan. The Levenberg-Marquardt Algorithm [J]. Tutoral on Lm Algorithm. 11(1):101110(2004).

[11] H. Zhou, Y. Yuan, C. Shi. Object tracking using SIFT features and mean shift [J]. Computer Vision \& Image Understanding. 113(3):345-352(2009).

[12] O. Chum, J. Matas. Optimal Randomized RANSAC [J]. IEEE Transactions on Pattern Analysis \& Machine Intelligence. 30(8):1472-1482(2007). 